\title{
VLA Observations of water maser emission associated with SVS 13
}

Luis F. Rodríguez

Instituto de Astronomía, UNAM, Campus Morelia, Apdo. Postal 3-72, Morelia, Michoacán 58089, México

Guillem Anglada

Instituto de Astrofísica de Andalucía, CSIC, Camino Bajo de Huétor 24, E-18008 Granada, Spain and Harvard-Smithsonian Center for Astrophysics, Cambridge, MA 02138, USA

José M. Torrelles

Institut d'Estudis Espacials de Catalunya (IEEC/CSIC) and Instituto de Ciencias del Espacio (CSIC), Edifici Nexus, c/ Gran Capità 2-4, E-08034 Barcelona, Spain

J. Eduardo Mendoza-Torres

Instituto Nacional de Astrofísica, Optica y Electrónica, Luis Enrique Erro No. 1, Apdo. Postal 51 y 216, 72840 Tonantzintla, Puebla, México

Aubrey D. Haschick

MIT Haystack Observatory, Westford, MA 01886, USA

Paul T. P. Ho

Harvard-Smithsonian Center for Astrophysics, Cambridge, MA 02138, $U S A$

Abstract. The young stellar object SVS 13 has been proposed as the powering source of the $\mathrm{HH} 7-11$ objects. Recent observations have revealed that in the radio continuum $(3.6-\mathrm{cm})$ the source is a binary separated by about 0.3 in the east-west direction. We present Very Large Array observations, made in the highest angular resolution $\mathrm{A}$ configuration, of $\mathrm{H}_{2} \mathrm{O}$ maser emission toward this source. Our data show that the $\mathrm{H}_{2} \mathrm{O}$ spots appear to be segregated in two velocity groups: a group with radial velocity similar to that of the ambient cloud $\left(V_{L S R} \simeq 8 \mathrm{~km}\right.$ $\mathrm{s}^{-1}$ ) that is associated with the western radio source, and a blueshifted (by $\sim 20 \mathrm{~km} \mathrm{~s}^{-1}$ ) velocity group that is associated with the eastern radio source. We discuss the possible implications of this observation. 


\section{Introduction}

HH 7-11 is a classical system of bright Herbig-Haro objects, first reported by Herbig (1974) and by Strom, Grasdalen, \& Strom (1974). Strom, Vrba, \& Strom (1976) discovered a $2.2 \mu \mathrm{m}$ source, SVS 13, roughly aligned with the chain of $\mathrm{HH}$ objects, that was proposed as their exciting source. Rodríguez, Anglada, \& Curiel (1997), through a high angular resolution VLA map of the region detected SVS 13 as a radio cm source (VLA 4), but also detected a new object, VLA 3, located $6^{\prime \prime}$ to the SW, and argued that this new object was a better candidate to drive the outflow. Deep radio maps with subarcsecond angular resolution obtained by Anglada, Rodríguez \& Torrelles (2000) revealed that the radio source associated with SVS 13 (VLA 4) is actually a binary (VLA 4A and $4 \mathrm{~B}$ ), separated by 0.3 , corresponding to a projected separation of $65 \mathrm{AU}$ for a distance of $220 \mathrm{pc}$.

The source SVS 13 presents a number of peculiar properties. It was detected initially as a near-IR source (Strom et al. 1976). Later, Goodrich (1986) detected a faint visible counterpart. The source exhibited a large increase of its brightness in the optical and near-IR in 1988-1990 (Eislöffel et al. 1991). Since then, the flux remained almost steady (Aspin \& Sandell 1994). In addition, despite being optically visible, SVS13 is a strong mm source (Grossman et al. 1987; Chini et al. 1997; Bachiller et al. 1998; Looney, Mundy, \& Welch 2000). Anglada et al. (2000) have argued that the two components of this binary system could be in different evolutionary stages and/or suffer from different extinction, with the more extincted object (proposed to be VLA 4B by Anglada et al.) being associated with the previously reported $\mathrm{mm}$ source, while the other component (VLA 4A) would correspond to the visible star SVS 13.

In order to further investigate SVS13, we have analyzed VLA observations of the $\mathrm{H}_{2} \mathrm{O}$ maser emission at $1.3 \mathrm{~cm}$ toward SVS 13 made with subarcsec angular resolution along several epochs. Our goals were to establish accurately the position of the $\mathrm{H}_{2} \mathrm{O}$ masers with respect to the components of the radio binary and to study if this relative location could shed light on the evolutionary stage of the stars.

\section{Observations and Discussion}

The $\mathrm{H}_{2} \mathrm{O}$ maser observations were made with the Very Large Array (VLA) of $\mathrm{NRAO}^{1}$ in the A configuration during 1983 October 21, 1985 February 18 , and 1988 October 31 . The phase calibrator was $0333+321$, for which we assumed a flux density of $1 \mathrm{Jy}$ at $1.3 \mathrm{~cm}$. The data were calibrated, edited, and imaged using the software AIPS of NRAO. We detected a total of nine $\mathrm{H}_{2} \mathrm{O}$ spots associated with SVS 13 over the three epochs (one in 1983, four in 1985, and four in 1988). Both in 1985 and in 1988 two spots at different radial velocity (with LSR radial velocities of -20.1 and $-15.5 \mathrm{~km} \mathrm{~s}^{-1}$ for 1985 and with LSR radial velocities of -14.8 and $-5.6 \mathrm{~km} \mathrm{~s}^{-1}$ for 1988, see Figure 1) appeared to coincide spatially within our relative positional uncertainty of $\sim 0$ "'02 and were

\footnotetext{
${ }^{1}$ The National Radio Astronomy Observatory is a facility of the National Science Foundation operated under cooperative agreement by Associated Universities, Inc.
} 


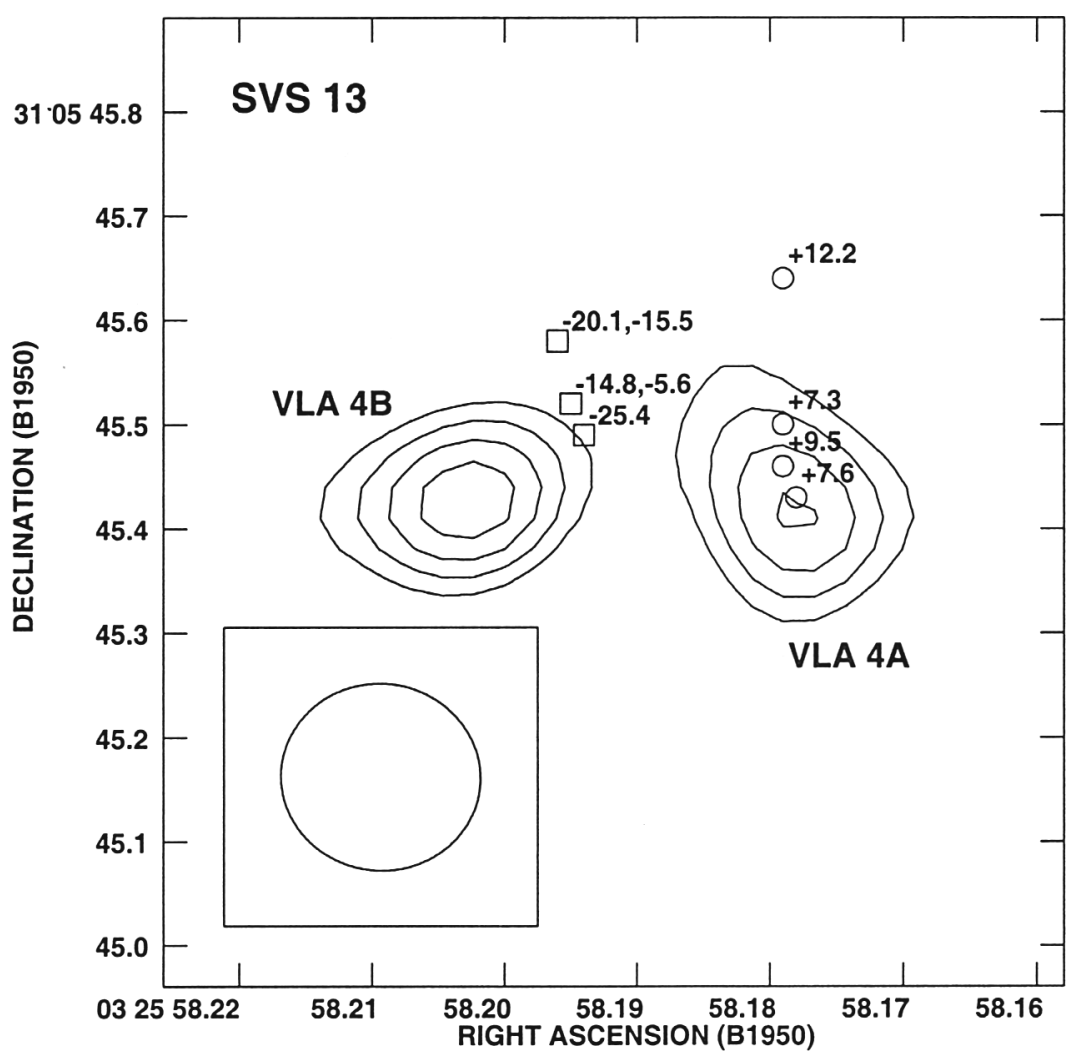

Figure 1. Position of the $\mathrm{H}_{2} \mathrm{O}$ spots overlapped in the $3.6 \mathrm{~cm}$ continuum image of Anglada et al. (2000). The LSR velocity of the spots is given in the figure. The contours of the continuum emission are $-3,3$, 4,5 , and 6 times $14 \mu \mathrm{Jy}$ beam $^{-1}$, the $\mathrm{rms}$ noise of the map. The half power contour of the beam is shown in the bottom left corner. Note that the spots with radial velocity close to that of the ambient cloud $\left(V_{L S R} \simeq 8 \mathrm{~km} \mathrm{~s}^{-1}\right)$ appear spatially associated with VLA $4 \mathrm{~A}$, while those with blueshifted radial velocity appear spatially associated with VLA 4B.

considered as arising from the same position (that was taken to be the average of the two nearby positions). We did not detect $\mathrm{H}_{2} \mathrm{O}$ emission in association with VLA 3, the source proposed by Rodríguez et al. (1997) as an alternative candidate to drive the $\mathrm{HH}$ 7-11 objects. In order to accurately compare the $\mathrm{H}_{2} \mathrm{O}$ maser positions with the $3.6 \mathrm{~cm}$ continuum positions of Anglada et al. (2000), that also used $0333+321$ as phase calibrator, we shifted the maser positions by $\Delta \alpha=+0.0047$ and $\Delta \delta=-0 . \prime 009$ to account for a recent small refinement of the position of $0333+321$.

In Figure 1 we show the maser spots overlapped on the $3.6 \mathrm{~cm}$ contour map of Anglada et al. (2000). As can be seen, the spots appear to be segregated in 
two velocity groups: a velocity group with $V_{L S R}$ similar to that of the ambient cloud $\left(V_{L S R} \simeq 8 \mathrm{~km} \mathrm{~s}^{-1}\right)$, for which we detected four features with $V_{L S R}$ in the range of 7.3 to $12.2 \mathrm{~km} \mathrm{~s}^{-1}$ ) and a blueshifted velocity group (five features with $V_{L S R}$ in the range of -25.4 to $-5.6 \mathrm{~km} \mathrm{~s}^{-1}$ ). Remarkably, all the spots in the ambient velocity group are associated with VLA 4A, while all the spots in the blueshifted velocity group are associated with VLA 4B.

We believe that this result supports the suggestion of Anglada et al. (2000) that there are at least two stars, VLA 4A and VLA 4B, in association with SVS 13. If the mean radial velocity of the maser groups were indicative of the radial velocity of the stars, one could argue that VLA $4 \mathrm{~A}$ is the more massive of the two (since its velocity is closer to the ambient cloud velocity) and that the blueshifted velocity seen in VLA $4 \mathrm{~B}$ indicates orbital motion for this star. However, at a distance of 220 pc (Cernis 1990), the angular separation between VLA $4 \mathrm{~A}$ and VLA $4 \mathrm{~B}$ corresponds to $\sim 70 \mathrm{AU}$, and a mass of $\sim 30 M_{\odot}$ would be required for VLA $4 \mathrm{~A}$. The modest bolometric luminosity of the source $\left(\sim 20 L_{\odot}\right.$; Jennings et al. 1987) seems to rule out such a massive star. We then tentatively propose that the radial velocity of both stars is most probably close to that of the ambient cloud, and that the blueshift observed in the $\mathrm{H}_{2} \mathrm{O}$ masers associated with VLA 4B are produced by outflow motions that are not present or are not as important in VLA 4A. VLBA monitoring of these masers, such as that reported by Wootten et al. (2001, these proceedings) should help clarify their nature.

\section{References}

Anglada, G., Rodríguez, L.F., \& Torrelles, J. M. 2000, ApJ, 542, L123

Aspin, C., \& Sandell, G. 1994, A\&A, 288, 803

Bachiller, R., Guilloteau, S., Gueth, F., Tafalla, M., Dutrey, A., Codella, C., \& Castets, A. 1998, A\&A, 339, L49

Černis, K. 1990, Ap\&SS, 166, 315

Chini, R., Reipurth, B., Sievers, A., Ward-Thompson, D., Haslam, C.G.T., Kreysa, E., \& Lemke, R. 1997, A\&A, 325, 542

Eislöffel, J., Günther, E., Hessman, F.V., Mundt, R., Poetzel, R., Carr, J.S., Beckwith, S., \& Ray, T.P. 1991, ApJ, 383, L19

Goodrich, R.W. 1986, AJ, 92, 885

Grossman, E.N., Masson, C.R., Sargent, A.I., Scoville, N.Z., Scott, S., \& Woody, D.P. 1987, ApJ, 320, 356

Herbig, G.H. 1974, Draft Catalog of Herbig-Haro Objects, Lick Obs. Bull. No. 658

Jennings, R. E., Cameron, D. H. M., Cudlip, W., \& Hirst, C. J. 1987, MNRAS, 226,461

Looney, L.W., Mundy, L.G., \& Welch, W.J. 2000, ApJ, 529, 477

Rodríguez, L.F., Anglada, G., \& Curiel, S. 1997, ApJ, 480, L125

Strom, S.E., Grasdalen, G.L., \& Strom, K.M. 1974, ApJ, 191, 111

Strom, S.E., Vrba, F.J., \& Strom, K.M. 1976, AJ, 81, 314 